\title{
A case of intrauterine fetal demise with cord prolapse
}

\section{Thulasi Devi, Gurusamy Prasad*}

Department of Obstetrics and Gynecology, Military Hospital, Thiruvananthapuram, Kerala, India

Received: 24 March 2021

Accepted: 29 April 2021

\section{*Correspondence:}

Dr. Gurusamy Prasad,

E-mail:mgrprasad7@gmail.com

Copyright: $\odot$ the author(s), publisher and licensee Medip Academy. This is an open-access article distributed under the terms of the Creative Commons Attribution Non-Commercial License, which permits unrestricted non-commercial use, distribution, and reproduction in any medium, provided the original work is properly cited.

\section{ABSTRACT}

A case of intrauterine fetal demise with cord prolapse, presenting to the labour room in active labour. Labour was uneventful; however, it reminds of importance of institutional deliveries, alert midwives, and importance of dedicated obstetric operation theatres and teams to prevent adverse maternal and neonatal outcome.

Keywords: Fetal hypoxia, Fetal demise, Obstetric emergencies

\section{INTRODUCTION}

Umbilical cord prolapse (UCP) is an acute obstetric emergency. An umbilical cord prolapse occurs when the amniotic membrane ruptures and the umbilical cord falls down from its usual position and exits the uterine cavity before the presenting part of the foetus, associated with an increased risk of perinatal mortality.

The mechanism of fetal demise is through near total or total acute asphyxia, which occurs when the umbilical cord becomes compressed as it lies ahead or alongside the fetal presenting part. The management of cord prolapse has undergone significant changes over the last century, and is now managed aggressively with 'immediate knife down' leading to improved maternal and fetal outcomes. ${ }^{1}$ There are two types of umbilical cord prolapse.

The first is overt prolapse, in which the cord prolapses in advance of the fetal presenting part and is palpable within the vagina or even visible from the vagina as in our case. In contrast, if the cord presents alongside the fetal presenting part but not below it, it is referred to as occult prolapse. The cord is not visible or palpable in occult prolapse. If the membranes are intact and cord is the presenting part then pulsations of cord are felt through the dilated internal os and is called cord presentation. It is in indication for caesarean section.

\section{CASE REPORT}

32-year-old $\mathrm{G}_{4} \mathrm{P}_{2} \mathrm{~L}_{2} \mathrm{~A}_{1}$, lady with previous two spontaneous vaginal delivery by midwife without any intrapartum and postpartum complications presented to us in active labour as unbooked, unregistered case, at around 38 weeks POG with history of spontaneous rupture of membranes around 48 hours back, loss of fetal movements with intrauterine fetal demise and cord prolapse (Figure 1).

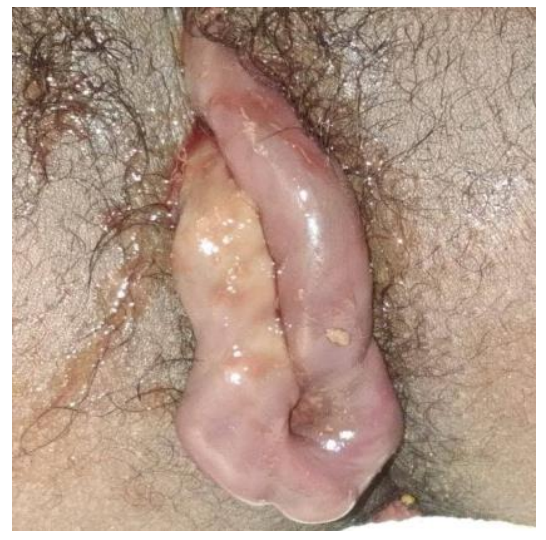

Figure 1: Cord lying in perineum outside interoitus, cord prolapse with IUFD. 
She was in advanced labour with good uterine contractions, cervix approximately $60 \%$ effaced, $6-7 \mathrm{~cm}$ dilated, membranes were absent and labour was augmented with oxytocin. She delivered still born male baby with mild signs of maceration uneventfully without any assistance or malpresentation. Liqour was meconium stained. Placenta and membranes were delivered in toto and her postpartum period was uneventful.

Routine antenatal investigations were done post-deliver, all biochemical and haematological parameters were WNL.

\section{DISCUSSION}

Spontaneous UCP can occur in otherwise uncomplicated pregnancies. These spontaneous causes share common characteristics: they are associated with fetal or maternal conditions that prevent the fetus from being engaged in the pelvis or because of abnormalities of the umbilical cord. Among the spontaneous risk factors, fetal malpresentation is the commonest as it is unlikely to be engaged in the maternal pelvis, thus allowing space for cord prolapse. ${ }^{2}$ Multiple gestation because of the increase in abnormal lie, polyhydramnios is also associated with unstable lie with fetal anomalies, both of which are also spontaneous risk factors. ${ }^{2,3}$ Other factors that increase the risk of UCP are preterm delivery, fetal growth restriction, or a birth weight less than 2500 g. ${ }^{4}$ Some other risk factors for cord prolapse include male fetal sex, umbilical cord length greater than $75 \mathrm{~cm}$, abnormal placentation, and a marginal cord insertion. ${ }^{5}$ Spontaneous rupture of membranes and preterm premature rupture of membranes are also independent risk factors. Most cases of UCP occur immediately after rupture of membranes, due to undiagnosed cord presentation or sudden decompression of uterus leading to prolapse of cord.

Intrapartum interventions that are considered benign and routine for labour management have been included in iatrogenic risk factors for UCP. They share one of two traits: they are either related to interventions that may cause the fetal presenting part to be elevated out of the pelvis or occur during ARM. These interventions include artificial rupture of membranes (especially if the fetal presenting part is not engaged), attempted rotation of the fetal head, amnioinfusion, external cephalic version in a patient with ruptured membranes, placement of an intrauterine pressure catheter or fetal scalp electrode, or placement of a cervical ripening balloon catheter. Inspite of a large percentage of cases of UCP being related to medical interventions, these iatrogenic causes are thought not to increase the associated perinatal morbidity and mortality because they are nearly always performed only on the labour and delivery unit, where the patient is likely undergoing continuous external fetal monitoring and where an urgent or emergent caesarean delivery can rapidly be performed. Many of the iatrogenic procedures are interventions that are used to treat spontaneous risk factors, and there is no evidence that these increase the incidence of umbilical cord prolapse. Murphy and MacKenzie note that the incidence of UCP is stable across populations, with no notable difference accountable to changes in obstetric practices. ${ }^{6}$

A large population-based retrospective cohort study including over 10 million births and identifying 16,126 cases of cord prolapse reported an increased incidence of placental abruption, excessive bleeding, premature rupture of membranes, and meconium-stained amniotic fluid in women who had a cord prolapse in labour. Of interest, this study also found that infants delivered via vaginal delivery after cord prolapse had a lower incidence of birth injury than infants delivered via caesarean delivery. This retrospective study could not identify an explanation for this reduction in birth injuries; however, it is speculated that the emergent nature of a caesarean delivery for cord prolapse could be more traumatic than a vaginal delivery. ${ }^{7}$ This reduction in birth injuries contrasts other studies that have shown caesarean delivery to be the safest, and therefore, it is the recommended mode of delivery in this emergent situation. ${ }^{8}$ Perinatal outcomes depend on a number of factors, which include gestational age, duration of cord prolapse before intervention, stage of labour, and level of the neonatal intensive care unit. $^{9}$ The perinatal mortality rate was significantly increased by cases of cord prolapse that occurred outside of the hospital and, therefore, prolonged the diagnosis-to-delivery interval. One study found that the largest independent risk factors for perinatal morbidity were grand multiparity and lack of antenatal care. Lack of antenatal care decreases identification of high-risk pregnancies and the ability to monitor these pregnancies accordingly. ${ }^{10}$ However, a study by Khan et al suggested that elevating the fetal presenting part and relieving cord compression were just as important as the interval to delivery. Maternal morbidity after a cord prolapse is most commonly due to complications of postpartum haemorrhage, as risk factors for postpartum haemorrhage and cord prolapse often overlap. Bladder injury resulting from back filling the maternal bladder to elevate the presenting fetal part and relieve cord compression has been reported. Abdominal wound infections despite the use of prophylactic antibiotics before caesarean delivery have been reported. This is likely due to the expedited manner in which these deliveries occur, possibly compromising the sterile field. ${ }^{11}$

The perinatal mortality rate in cases of cord prolapse has decreased substantially after the move toward emergent caesarean delivery after diagnosis. A retrospective cohort study from the National Maternal Hospital in Dublin, which spanned a 69-year period, showed a significant increase in perinatal survival rates from $46 \%$ in 1940 to $94 \%$ in 2009 . The study also reported improvement in perinatal morbidity in perinatal encephalopathy occurring in $2 \%$ of cases with umbilical cord prolapse and cerebral palsy in $0.43 \% .^{12}$ The recommendation for caesarean delivery, decreased incidence of cord prolapse related to 
decreased incidence of grand multiparity, improved ultrasound diagnosis of fetal malpresentation, and aggressive neonatal resuscitation, accounts for improved outcomes. $^{13}$

If cord presentation is diagnosed in the intrapartum period after labour has begun but membranes are intact, amniotomy should be avoided unless it is indicated. Amniotomy, if indicated, should be performed under strict precautions if the fetal head is not well engaged. Controlled rupture of membranes by an experienced obstetrician is recommended. Amniotomy should be performed with a hypodermic needle or pudendal nerve trumpet to facilitate a slow, controlled drain of amniotic fluid. If cord presentation is diagnosed in the intrapartum period after rupture of membranes have occurred, reduction above the presenting part can be considered while making preparations for emergent caesarean delivery; however, reduction is rarely performed and has been largely abandoned except to elevate the presenting part till expeditious delivery. ${ }^{12}$

Manoeuvres which can be performed and are mentioned in various textbooks are enumerated below: (a) Manual elevation of presenting fetal part: The fetal head can be elevated by upward force with fingers or hand in the vagina, if tolerated. When elevating the presenting fetal part manually, it is ideal not to manipulate the cord as this can induce vasospasm in the umbilical vessels. The patient can be positioned in steep trendelenburg or positioned in the knee-chest position to relieve compression of the cord. (b) Filling the bladder: Using a Foley's catheter, the bladder is filled with $500 \mathrm{~mL}$ or more fluid, which elevates the presenting fetal part and supposedly relieves cord compression. This method, first described by Vago in 1970 , proposes that the bladder be filled until the bladder is seen as swelling above the pubis for optimal elevation of the fetal part. ${ }^{14}$ It has also been noted to decrease uterine contractions and assist in relieving cord compression. ${ }^{15} \mathrm{~A}$ study by Bord et al compared perinatal outcomes of cord prolapse cases in which bladder filling was used in addition to supporting the presenting part with a vaginal hand and cases where only a vaginal hand was used for support. This study found there were no significant differences in neonatal outcomes between the two groups. ${ }^{16}$ (c) Tocolytics: The use of tocolytics during the interval from diagnosis to delivery has been described to decrease contractions and, therefore, decrease pressure on the umbilical cord. ${ }^{17}$ There is a risk of causing uterine atony after delivery, and therefore, tocolytics should be considered if the interval until delivery is expected to be delayed. Tocolytics should not be considered a primary management tool. ${ }^{15}$ (d) Cord replacement or reduction: Cord replacement or reduction was the initial step in management of cord prolapse from the 1920s until caesarean delivery was advocated for such emergencies. ${ }^{18}$ The technique of cord replacement as management has been described before caesarean delivery became the standard for delivery after a cord prolapse, and is not encouraged. (e) Diagnosis-to-delivery interval: Optimal outcome has been described when the interval from diagnosis to delivery is less than 30 minutes. The prevention of fetal neurologic injury in cases of umbilical cord prolapse may depend on more than a cut off of a 30minute decision-to-delivery interval. If prolonged fetal heart rate decelerations last 3 minutes or less, there is evidence in animal models that the foetus has the ability to recover and 30 minutes to delivery may not result in worse outcomes. However, if there is sustained fetal bradycardia for a longer period, the foetus may not be able to recover cerebral blood flow. If the ability to recover cerebral blood flow autoregulation is lost after 10 minutes of cord occlusion, then delivery before this may reduce the risk of a permanent neurologic injury. Although all cases should be treated emergently, a patient with sustained fetal bradycardia with a shorter interval to delivery may improve outcomes. (f) Prevent vasoconstriction/ vasospasm: Vasospasm of cord should be prevented by covering the exposed cord with warm saline soaked sterile abdominal swab if it cannot be reposited gently back into vagina and to cover the transportation interval to caesarean section. Minimal handling of cord should be done.

Routine antenatal ultrasound cannot predict cord prolapse. Ladies with malpresentation, unstable lie, multiple gestation, polyhydramnios should be encouraged to report to hospital as soon as possible in case of onset of labour or rupture of membranes. In case of high floating presenting part in absence of labour artificial rupture of membranes should be avoided or should be done in a controlled setting. Women should be counselled for continuation or termination at threshold of fetal viability, lethal congenital malformations and fetal demise in case of cord prolapse. Caesarean section is contraindicated in such cases. Immediate resuscitation of newborn takes priority over delayed cord clamping.

\section{CONCLUSION}

UCP is a rare occurrence and is a life-threatening emergency for the foetus. These events are unpredictable and unpreventable. UCP requires swift diagnosis and management for optimal outcome. The management is an expedited delivery with efforts to relieve cord compression until delivery can be achieved. Delivery should be performed as expeditiously as possible by caesarean delivery if vaginal delivery is not imminent. Several studies have shown that an interval to delivery of 30 minutes or less resulted in better neonatal outcomes compared with an interval greater than 30 minutes. Umbilical cord prolapse simulation training has been shown to improve outcomes. Organizations that conduct team training with simulations and drills can improve team work and communication and reduce adverse fetomaternal outcomes. Simulation drills should include all team members including, nursing staff, anaesthesiologist and obstetricians. Mechanism of fetal morbidity and mortality is severe birth asphyxia. 
Funding: No funding sources

Conflict of interest: None declared

Ethical approval: Not required

\section{REFERENCES}

1. Redline RW. Disorders of placental circulation and the fetal brain. Clin Perinatol. 2009;36(3):549-59.

2. Kahana B, Sheiner E, Levy A, Lazer S, Mazor M. Umbilical cord prolapse and perinatal outcomes. Int $\mathbf{J}$ Gynaecol Obstet. 2004;84(2):127-32.

3. Lin MG. Umbilical cord prolapse. Obstet Gynecol Surv. 2006;61(4):269-77.

4. Koonings PP, Paul RH, Campbell K. Umbilical cord prolapse. A contemporary look. J Reprod Med. 1990;35(7):690-2.

5. Adegbola $\mathrm{O}$, Ayanbode $\mathrm{O}$. The incidence, risk factors and determinants of perinatal outcome of umbilical cord prolapses in Lagos, Nigeria. Niger Med J. 2017;58(2):53-7.

6. Murphy DJ, Kenzie MIZ. The mortality and morbidity associated with umbilical cord prolapse. Br J Obstet Gynaecol. 1995;102(10):826-30.

7. Behbehani S, Patenaude V, Abenhaim HA. Maternal Risk Factors and Outcomes of Umbilical Cord Prolapse: A Population-Based Study. J Obstet Gynaecol Can. 2016;38(1):23-8.

8. Hasegawa J, Sekizawa A, Ikeda T, Koresawa M, Ishiwata I, Kawabata M, et al. Clinical risk factors for poor neonatal outcomes in umbilical cord prolapse. $\mathrm{J}$ Matern Fetal Neonatal Med. 2016;29(10):1652-6.

9. Enakpene CA, Odukogbe AT, Morhason BIO, Omigbodun AO, Arowojolu AO. The influence of health-seeking behavior on the incidence and perinatal outcome of umbilical cord prolapse in Nigeria. Int $\mathrm{J}$ Womens Health. 2010;2:177-82.
10. Kahana B, Sheiner E, Levy A, Lazer S, Mazor M. Umbilical cord prolapse and perinatal outcomes. Int J Gynaecol Obstet. 2004;84(2):127-32.

11. Khan RS, Naru T, Nizami F. Umbilical cord prolapse-a review of diagnosis to delivery interval on perinatal and maternal outcome. J Pak Med Assoc. 2007;57(10):487-91.

12. Gibbons C, Herlihy C, Murphy JF. Umbilical cord prolapse--changing patterns and improved outcomes: a retrospective cohort study. BJOG. 2014;121(13):1705-8.

13. Nelson DB, Lucke AM, McIntire DD, Sánchez PJ, Leveno KJ, Chalak LF. Obstetric antecedents to bodycooling treatment of the newborn infant. Am J Obstet Gynecol. 2014;211(2):155.

14. Vago T. The management of prolapse of the umbilical cord. Am J Obstet Gynecol. 1994;170(5):1476.

15. Holbrook BD, Phelan ST. Umbilical cord prolapse. Obstet Gynecol Clin North Am. 2013;40(1):1-14.

16. Bord I, Gemer O, Anteby EY, Shenhav S. The value of bladder filling in addition to manual elevation of presenting fetal part in cases of cord prolapse. Arch Gynecol Obstet. 2011;283(5):989-91.

17. Sayed AWA, Hamdy MA. Optimal management of umbilical cord prolapse. Int $\mathrm{J}$ Womens Health. 2018;10:459-65.

18. Barrett JM. Funic reduction for the management of umbilical cord prolapse. Am J Obstet Gynecol. 1991;165(3):654-7.

Cite this article as: Devi LT, Prasad G. A case of intrauterine fetal demise with cord prolapse. Int J Reprod Contracept Obstet Gynecol 2021;10:2537-40. 